\title{
La Red Internacional de Investigación Ecológica a Largo Plazo (ILTER) a 20 años de su creación: sus avances y retos
}

\author{
The International Long Term Ecological Research (ILTER) \\ network after 20 years since its creation: advances and challenges
}

\author{
Manuel Maass $^{\text {a*, }}$, Miguel Equihua ${ }^{b}$ \\ *Autor de correspondencia: ${ }^{a}$ Universidad Nacional Autónoma de México (UNAM Campus-Morelia), \\ Centro de Investigaciones en Ecosistemas (CIEco), Michoacán, México, tel.: +52 (443) 322-2701, maass@cieco.unam.mx \\ ${ }^{\mathrm{b}}$ Instituto de Ecología A.C. (INECOL), Xalapa, Veracruz, México.
}

\begin{abstract}
SUMMARY
Twenty years after being established, the ILTER network embraces more than 600 groups from 37 countries. The ecological research at the ILTER network has moved from a merely ecological focus toward a more socio-ecological approach. The network is now more committed to social needs and with higher international presence. An important challenge in the next years will be to encourage a more socioecosystem and trans-disciplinary research. Its wide and flexible research agenda, along with its particular commitment from its groups to work on specific sites for a long time (know as "site based research"), makes ILTER particularly useful and relevant in dealing with the current environmental crisis. The network has incorporated tools and protocols to improve collaboration, such as common metadata management protocols and a multilingual information research system. It is still necessary to improve the efficiency of information delivery to the general public, promoting a more integrated, useful, comprehensive and local context information. The network should also keep promoting "bottom up" initiatives as well as the participation of students and early career scientists. We cannot expect the same level of participation between networks. However, we do expect the same level of commitment and should promote the evaluation of the national networks; not as an exclusion process, but as a mechanism to stimulate collaboration, as well as for an early detection of problems of integration. The ILTER network should keep increasing its geographic presence promoting the integration of single affiliated sites as seeds for the development of future national networks.
\end{abstract}

Key words: LTER, ILTER, socioecosystem, socio-ecological systems, transdiscipline, Mex-LTER.

\section{RESUMEN}

La red Internacional de Investigación Ecológica a Largo Plazo (ILTER), a sus 20 años de creación, acoge a cerca de 600 grupos de académicos de 37 países. La red ha ido migrando de una investigación estrictamente ecológica a una que incluye aspectos socioambientales, más comprometida, relevante y con mayor presencia internacional. La red ILTER no está sola en el esfuerzo por comprender y ayudar a resolver los problemas ambientales, pero su amplia y versátil agenda de investigación, aunado al peculiar compromiso de trabajar en sitios específicos durante décadas, hace a ILTER particularmente útil y relevante. Un reto importante será incorporar investigación de corte socioecosistémico y transdisciplinario. Se han desarrollado protocolos comunes de manejo de metadatos y cuenta con un sistema de búsquedas de información multi-lingual. Además, es necesario mejorar la entrega de información a la sociedad para hacerlo de manera más eficiente, promoviendo que esta sea integrada, útil, oportuna, comprehensiva y localmente pertinente. Se deberá seguir impulsando la contribución desde los cuerpos académicos e incentivar mayor colaboración estudiantil. No se puede esperar el mismo nivel de participación entre todos sus miembros, pero sí el mismo nivel de compromiso, por lo que se deberá estimular la evaluación del desempeño de las redes nacionales, no como mecanismo de exclusión sino de estímulo y atención oportuna a problemas de colaboración. ILTER deberá seguir expandiendo su presencia geográfica, particularmente en países en vías de desarrollo, estimulando la participación de un mayor número de grupos académicos, como estrategia para promover la construcción de nuevas redes nacionales.

Palabras clave: LTER, socioecosistemas, sistemas socio-ecológicos, transdisciplina, Mex-LTER.

\section{INTRODUCCIÓN}

La red Internacional de Investigación Ecológica de Largo Plazo, conocida como ILTER, por sus siglas en inglés, cumplió 20 años. Los aniversarios invitan a hacer una pausa en el camino y revisar lo que se está haciendo bien para continuar haciéndolo, e identificar lo que se está haciendo mal para corregirlo. La red ILTER (2014) tiene como visión; un mundo en el que la ciencia ayuda a prevenir y resolver los problemas socio-ecológicos, y tiene como misión mejorar el entendimiento del ecosistema global e informar a la sociedad sobre sus problemas ambientales actuales y futuros, utilizando una estrategia de trabajo en red de grupos académicos anclados en sitios particula- 
res y realizando investigación ecológica y socioeconómica de largo plazo.

México se incorporó a la Red Internacional en 2002. A lo largo de estos años el aprendizaje se ha realizado en un proceso de manejo adaptativo, el arduo reto del trabajo en red. Así mismo se ha participado de la evolución de la red ILTER, la cual ha ido migrando de a) una investigación principalmente ecológica a una más de corte socio ambiental; b) de una estrategia de trabajo dirigida desde arriba (los comités y coordinadores nacionales), a una más orientada a identificar los intereses de la base (los grupos académicos y los investigadores); c) de una situación relativamente desconectada entre redes nacionales, a una conectividad más clara entre sus miembros; d) de poca visibilidad, a una mayor presencia internacional; e) de una ciencia estimulada por la curiosidad, a una ciencia más comprometida y socialmente pertinente, y f) de un formato similar al de las sociedades científicas, a uno más complejo de trabajo en red y de colaboración académica. La red ha crecido a razón de dos países por año con un promedio de 30 sitios por año. Su reuniones han pasado de ser de tipo organizacional (básicamente para resolver asuntos administrativos), a reuniones más científicas en las que se discute la investigación de los países anfitriones y se estimulan los trabajos de colaboración académica internacional.

No obstante lo anterior, es importante reconocer que existen algunas deficiencias que habrá que resolver en los próximos años. Así mismo, existen importantes retos a enfrentar como red, a fin de cumplir cabalmente con nuestra misión y objetivos. En las próximas líneas se hace un breve recuento de estos logros y retos a fin de capitalizar la experiencia adquirida, así como para proporcionar una mirada de hacia dónde debería ir la Red en sus próximos 10 años.

\section{COLABORACIÓN}

La red ILTER no está sola en el esfuerzo por comprender y ayudar a resolver los problemas ambientales. Existen múltiples organizaciones de corte internacional con una extensa gama de intereses, marcos conceptuales y formatos de operación. Lo que hace a la red ILTER particularmente útil y relevante en este esfuerzo es el compromiso de sus grupos académicos de trabajar anclados en sitios específicos (site-based research) durante mucho tiempo (long-term research). Esto no solo permitió ir acumulando un entendimiento cada vez más profundo de los procesos ecológicos y de sus vínculos con la dinámica social de los sitios de estudio, sino que además permitió ir generando procesos de construcción de confianza y comprensión mutua entre los científicos y las comunidades locales, como un ingrediente indispensable en el trabajo de investigación participativa (por ejemplo, colaborando en la detonación de programas de "gestión integrada de cuencas", desarrollando proyectos de "ciencia ciudadana", organizando eventos de "puerta abiertas", etc.). La red ILTER ha ido evolucionando de una investigación ecológica de corte multidisciplinario, a una red socio-ecológica de corte más interdisciplinario. El reto para los próximos años es ir aún más allá y convertirla en una red de investigación socioecosistémica de corte verdaderamente transdisciplinario. Este no será un paso fácil pues implica reconocer al ser humano como un fenómeno eminentemente físico-químico-biológico-humano inseparable (Maass 2012). Con este enfoque, los socioecosistemas se conciben como la interacción de componentes abióticos, bióticos y humanos de manera integrada a diferentes escalas de tiempo y espacio. El enfoque socioecosistémico se plantea como un nuevo paradigma en la ciencia, con implicaciones epistemológicas, metodológicas, éticas e institucionales muy importantes (Maass 2012). Es por ello que se habla de enfoques transdisciplinarios en los que, de acuerdo con Spangenber (2011), la fuente de inteligencia para el manejo sustentable de socioecosistemas se extiende a incluir el conocimiento no científico, de tal forma que las preguntas de investigación se definen de manera conjunta entre científicos y otros miembros de la sociedad y la calidad del trabajo es revisada por ambos grupos, los usuarios como expertos en relevancia, y los científicos como expertos en rigor.

Programas internacionales como el PECS (Program of Ecosystem Change and Society), han incorporado este enfoque socioecosistémico y transdisciplinario (Carpenter et al. 2012), en un intento por continuar con los esfuerzos iniciados en el Millenium Ecosystem Assesment, pero tratando de subsanar el problema con el que se confrontó al encontrar una ciencia muy fragmentada en la que incluso los científicos más involucrados en investigación interdisciplinaria no suelen cruzar las fronteras territoriales de sus campos de trabajo (Norgaard 2008). Este programa para el estudio del cambio de los ecosistemas y la sociedad es auspiciado por el Consejo Internacional para la Ciencia (ICSU) y forma parte de la iniciativa Future Earth como una estrategia de investigación para lograr la sustentabilidad global. Tal y como está sucediendo con otras iniciativas internacionales, la participación de la ILTER en este esfuerzo internacional no solamente será muy oportuna, sino que además le dará una gran visibilidad.

\section{TRABAJO EN RED}

El trabajo en red dentro de la academia, va más allá del trabajo típico de las sociedades científicas ya que este busca fomentar la interacción y el trabajo colaborativo entre sus miembros. No sólo se trata de compartir hallazgos con el resto de los colegas sino de identificar problemas de interés común que no pueden ser resueltos de manera individual. Es por ello que el diseño, la planeación y la implementación del esfuerzo para alcanzar el objetivo se hace de manera conjunta y, por tanto, las reglas del juego del quehacer científico cambian radicalmente. En el trabajo en red lo que se busca es colaborar más y competir menos. Se propicia el encuentro de científicos para compartir habilidades y se evita la segregación de acuerdo a las ca- 
pacidades. En el trabajo en red se promueve la discusión y se evita la confrontación; se incentiva la creatividad como camino hacia la originalidad; y se privilegia el reconocimiento colectivo más que el individual. Se promueve la diversidad y se aprecia el lado positivo de la disidencia, sospechando de los consensos alcanzados sin debate. Todo esto hace que el trabajo en red exija de la academia nuevas aproximaciones para colaborar eficazmente.

De igual forma, y en este contexto de la ciencia interdisciplinaria, el trabajo en red debe extenderse a incluir tanto académicos de las ciencias naturales como a los de las ciencias sociales. El reto es aún mayor cuando se busca un trabajo verdaderamente transdisciplinario, en el que las redes académicas deben crecer para lograr un vínculo con redes de otros sectores no académicos, tales como las de los empresarios, los productores, las ONG, etc.

\section{COMUNICACIÓN}

La comunicación es central en todas las redes, no sólo para mantener en contacto a sus miembros sino también como una herramienta de interacción con otros sectores sociales. El tema del manejo de información, es quizá una de los más desarrollados en la red ILTER. No sólo se cuenta con un excelente portal en la web, sino además se ha construido un formato y un protocolo común de manejo de metadatos (EML, por sus siglas en inglés: Ecological Metadata Language). Actualmente se cuenta con un sistema de búsquedas de información de corte multilingual basado en la tecnología informática de ontologías que permite identificar conceptos y datos asociados con ellos con gran precisión, independientemente de diferencias de idioma e incluso culturales. En la red ILTER se ha adoptado el Drupal Ecological Information Management System (DEIMS). Se cuenta también con una herramienta de cómputo que permite buscar información característica de los grupos académicos de la red, de tal manera que es posible identificar, por ejemplo: sitios ubicados entre pares de coordenadas geográficas o sitios con una misma altitud pero con diferente precipitación.

Un aspecto en el que se debe trabajar más es el de incorporar mayor información en estos sistemas de búsqueda. Desde hace varios años se ha insistido en que la información pertinente para la toma de decisiones no sólo necesita estar disponible para todos los sectores sociales potencialmente interesados, sino además debe ser entregada en forma eficiente, entendido por ello que la información esté integrada, que sea útil, que sea oportuna, comprehensiva y localmente relevante (Vaughan et al. 2007).

Otro aspecto importante que está tomando fuerza es lo que se conoce como Big Data, considerado por algunos como "una revolución que transformará la manera como vivimos y pensamos" (Mayer-Schönberger y Cukier 2013). El concepto incluye los sensores, los geoposicionadores (GPS) en los celulares y los clicks que miles de millones de personas hacen en Google, Amazon, Facebook,
LikendIn y Twiter. Estos datos pueden ser analizados por las computadoras actuales con cada vez mayor facilidad. La red ILTER no puede dejar pasar la oportunidad de utilizar estas nuevas tecnologías y herramientas, pero para contar con información confiable, "datificarla" e interpretarla es necesario saber cómo y qué medir. Aspecto en el que los investigadores de la red son expertos.

También se quiere enfatizar la necesidad de lograr una mejor y mayor comunicación entre los representantes de las redes nacionales (que son los que asisten a las reuniones del Comité Coordinador), con los cuerpos académicos en la base (es decir, los investigadores de los sitios de la red). Un primer esfuerzo ha sido el armado de una base de datos con los miles de correos electrónicos de los investigadores individuales de la red (all scientist webmail list) a los que de manera periódica se les hace llegar información de interés general. Así mismo, ya se están planeando reuniones en las que se convoque a todos los investigadores (all scientists meetings) como sucederá en la reunión de Chile este año (2014), a la que se invitará a participar a todos los investigadores de las redes del continente americano.

Finalmente, es importante reconocer que la mejor manera de comunicarse entre los investigadores de la red y otros sectores sociales, es mediante el involucramiento de los académicos en la solución de problemas reales de manejo mediante estrategias de investigación participativa tales como en los procesos de "investigación-acción", el "co-diseño", el "manejo adaptativo", la "ciencia ciudadana" y el "monitoreo comunitario", por mencionar algunos enfoques que han estado apareciendo en la literatura recientemente (ver por ejemplo, Lynam et al. 2007, Sanders y Stappers 2008, Kofinas 2009 y Burgos et al. 2013).

\section{ENFOQUE DESDE LAS BASES}

Ligado con la sección anterior, la red ILTER deberá impulsar con mayor fuerza la estrategia que hace tres años implementó para fomentar la participación desde la base (bottom up approach), de tal forma que investigadores individuales pudieran promover el desarrollo de iniciativas y/o proyectos de corte internacional aprovechando la capacidad instalada de la red ILTER. La idea es facilitar la participación de los grupos simplificando lo más posible la información que se les pide a cada sitio, buscando las sinergias y los procesos de síntesis que surgen del trabajo colectivo, permitiendo así entender procesos ecológicos que operan a mayor escala. Ya ha habido casos muy exitosos, como la propuesta de Ricardo Díaz Delgado de la red Española, en el sentido de mapear las interacciones entre redes nacionales y seguirlas en el tiempo a fin de tener una película de cómo va fluctuando la colaboración entre redes y usar la información para identificar problemas de conectividad, para inducir procesos de trabajo en red y tener un mecanismo eficiente para ver los resultados del esfuerzo colectivo. También se puede citar el esfuerzo de Hideaki Shibata de la red Japonesa, quien ha fomentado la 
participación de otros miembros de la red en el marco de los trabajos del proyecto GLP (Global Land Project) para atacar preguntas tan complejas y actuales como: ¿cuáles son los procesos críticos, lo detonadores, los mecanismos de retroalimentación y los umbrales que causan un cambio abrupto en la socio-biogeoquímica del nitrógeno a nivel regional y global? De igual forma, se puede mencionar el proyecto de Melinda Smith y colaboradores, quienes han logrado comparar múltiples sitios de praderas en los Estados Unidos con praderas en Sudáfrica para investigar si la pérdida de grandes herbívoros afectan las comunidades de plantas. Todos estos proyectos tienen en común que fueron propuestos por el interés particular de un investigador miembro de la red y que para su implementación se utilizaron cantidades muy pequeñas de recursos económicos.

Otro proyecto de esta índole que es interesante describir con un poco más de detalle es la propuesta de Eeva Furman y Riikka Paloniemii de la red Finlandesa. Ellas le han propuesto a la red una iniciativa que intenta construir un foro ciudadano a través de la red global de Internet, para facilitar el diálogo entre científicos y la sociedad. La idea es, aprovechando la capacidad instalada de la ILTER (con sus más de 600 grupos académicos), encontrar grupos que se comprometan a identificar familias en su sitio de investigación, que estén interesadas en participar en un esfuerzo de largo plazo. Los investigadores realizarán encuestas esporádicas a los habitantes de las familias participantes, a fin de colectar datos sobre el comportamiento y las actitudes de la población respecto a problemas ambientales específicos. El nivel de recursos económicos que se requieren para montar esta iniciativa es relativamente bajo y el potencial de generar información de alta calidad es enorme.

\section{CONSTRUCCIÓN DE CAPACIDADES}

La Red tiene como otro de sus objetivos promover la creación de infraestructura de investigación en los sitios de estudio, así como mejorar las capacidades académicas a través de la formación de recursos humanos. Es claro que este objetivo se ha cumplido ampliamente en muchas de las redes nacionales que han visto crecer sus instalaciones y constatar que varios de los estudiantes vinculados con grupos de la red ahora se han convertido en investigadores activos en ella. No obstante la existencia de redes con un amplio programa de participación estudiantil (como es el caso de la red Americana de Investigación Ecológica de Largo Plazo, USLTER), la red ILTER apenas hace algunos años comenzó a incursionar en esto mediante cursos de verano promovidos y auspiciados por la red Japonesa. Será muy importante que en los próximos años la ILTER le dé un mayor impulso a la participación estudiantil en sus reuniones. Recientemente surgió una buena oportunidad con la creación de la INNGE (International Network of Next Generation Ecologists) que busca reunir a ecólogos de todo el mundo, que están iniciando su carrera académica (estudiantes graduados y recién egresados) con la esperanza de robustecer las ligas internacionales. Esta iniciativa surge en el marco de los trabajo de la INTECOL (Internacional Ecological Society) con quienes la ILTER ha colaborado ampliamente.

\section{EVALUACIÓN}

Existe una gran heterogeneidad entre los países miembros de la red ILTER. Como es de suponer, no se puede esperar el mismo nivel de participación entre todos sus miembros. Sin embargo, sí se debe esperar el mismo nivel de compromiso. En ese sentido, las actividades que se promueven en la red son de varios tipos, y se busca que ni las redes emergentes se constituyan en un lastre para las redes bien consolidadas, ni que estas últimas dejen atrás al resto. Ciertamente han aumentado los requisitos y el rigor con el que se evalúan las solicitudes de ingreso; sin embargo, en los próximos años se debe implementar un mecanismo de revisión de la participación y el desempeño de las redes. No como un mecanismo de exclusión competitiva, sino como un ejercicio de manejo adaptativo que nos permita estimular lo que se está haciendo bien, y responder oportunamente cuando se detecten problemas en el desempeño de las mismas.

\section{CRECIMIENTO}

A 20 años de creación, la red está entrando en una etapa de consolidación y no se espera que siga creciendo a razón de dos redes nacionales por año, como lo ha hecho desde su comienzo en 1993. Sin embargo, aún se espera la incorporación de varios países, incluyendo algunos con gran cobertura territorial como la India, Rusia y Argentina.

Dado que la filosofía de la red es ser lo más amplia e incluyente posible, se ha hecho un esfuerzo por diseminar el interés de investigación a largo plazo aún en países con pocos recursos económicos y humanos. No se trata de bajar los estándares, sino de crear las condiciones para que estos se puedan alcanzar más fácilmente. Se ha identificado la existencia de excelentes grupos académicos que realizan investigación ecológica con la filosofía y los estándares de la ILTER, pero que, por diversas razones, no ha logrado un proceso de conformación de una red nacional. Es por ello que se revisó la conveniencia de establecer un nuevo tipo de afiliación en el que se le dé cabida a estos grupos académicos. Dichos grupos afiliados a la red ILTER deberán contar con el respaldo y acompañamiento de alguna red miembro. La vigencia de su incorporación deberá ser renovada cada cinco años y tendrán el compromiso de participar en la construcción de sus redes nacionales. La implementación de esta estrategia nos permitirá expandir el ámbito de acción de la ILTER.

\section{CONSIDERACIONES FINALES}

Sólo resta enfatizar que, a pesar de que la red ILTER no cuenta con oficinas centrales ni un financiamiento importante, más allá de las esporádicas aportaciones económicas de algunos de sus países miembro, esta se ha mantenido ac- 
tiva y va progresando desde su creación en 1993, producto del esfuerzo comprometido y voluntario de sus miembros. Su Comité Coordinador, conformado por los representantes nacionales de cada red afiliada, se ha reunido anualmente de manera ininterrumpida por 20 años, y hoy en día cuenta con más recursos, más independencia y más procesos democráticos que en años anteriores. La red ha crecido significativamente de tal forma que hoy acoge a más de 600 grupos académicos de 39 países repartidos en los cinco continentes (ILTER 2014), todos ellos sintonizados en una misma visión y misión, y convencidos de una forma particular de hacer investigación científica, colectiva, asumiendo la convicción de mantener el esfuerzo por décadas, y anclados en sitios particulares de trabajo. Esto desde luego ha implicado una curva lenta de avance y aprendizaje, pues siempre será más fácil reunir a 200 científicos para exponer sus proyectos particulares, que juntar a 20 de ellos para formular una propuesta de trabajo colectivo. El esfuerzo bien ha valido la pena pues es precisamente este trabajo colectivo lo que permite alcanzar metas correspondientemente más difíciles y más complejas, que las que se alcanzan con esfuerzos individuales. El trabajo en red implica un cambio radical en las reglas del juego del quehacer científico. Reconocerlo y hacerlo con eficiencia es precisamente el gran reto de la ILTER.

\section{AGRADECIMIENTOS}

Agradecemos a Mariela Núñez-Ávila, Juan Armesto y Ricardo Rozzi por su invitación a colaborar en los trabajo de la red Chilena de Investigación Ecológica de Largo Plazo. Así mismo agradecemos a Raúl Ahedo, Atzimba López, Lyliana Rentería y Socorro Lara por su ayuda en la preparación del documento, así como a Tamara Contador y a un revisor anónimo por sus comentarios y sugerencias al mismo. Los Autores agradecemos también el apoyo que el Consejo Nacional de Ciencia y Tecnología de México (CONACYT) le ha brindado a la Red Mex-LTER. Este trabajo se terminó de escribir durante un estancia sabática del Dr. Manuel Maass en el Instituto Finlandés del Medio Ambiente (SYKE) con un apoyo de la DGAPA-UNAM.

\section{REFERENCIAS}

Burgos A, R Páez, E Carmona, H Rivas. 2013. A systems approach to modeling Community-Based Environmental Monitoring: a case of participatory water quality monitoring in rural Mexico. Environmental Monitoring and Assessment 185(12): 10297-10316.

Carpenter SR, C Folke, A Norstrom, O Olsson, L Schultz, B Agarwal, P Balvanera, B Campbell, JC Castilla, W Cramer, R DeFries, P Eyzaguirre, TP Hughes, S Polasky, Z Sanusi, R Scholes, M Spierenburg. 2012. Program on ecosystem change and society: an international research strategy for integrated social-ecological systems. Current Opinion in Environmental Sustainability 4: 1-5.

ILTER (International Long Term Ecological Research Network, CR). 2014. International Long Term Ecological Research Network. Disponible en: www.ilternet.edu

Kofinas GP. 2009. Adaptive co-management in social-ecological governance. In Principles of ecosystem stewardship. Nueva York, USA. Springer. p. 77-101.

Lynam T, W De Jong, D Sheil, T Kusumanto, K Evans. 2007. A review of tools for incorporating community knowledge, preferences, and values into decision making in natural resources management. Ecology and Society 12(1): 5.

Maass JM. 2012. El manejo sustentable de socio-ecosistemas. In Calva JL ed. Cambio climático y políticas de desarrollo sustentable. Análisis Estratégico para el Desarrollo, volumen 14. México DF, México. Juan Pablos Editor-Consejo Nacional de Universitarios. 395 p.

Mayer-Schönberger V, K Cukier. 2013. Big Data: a revolution that will transform how we live, work and think. Boston, USA. Eamon Dolan Book. 256 p.

Norgaard RB. 2008. Finding Hope in the Millennium Ecosystem Assessment: an Essay. Conservation Biology 22(4):862-869.

Sanders E, PJ Stappers. 2008. Co-creation and the new landscapes of design. International CoDesign 4: 5-18.

Spangenber JH. 2011. Sustainability science: a review, an analysis and some empirical lessons. Environmental Conservation 38: 275-287.

Vaughan H, RB Waide, JM Maass, E Ezcurra. 2007. Developing and delivering scientific information in response to emerging needs. Frontiers in Ecology and Environment 5(4): W8-W11. 
